\title{
Database Replication Using Generalized Snapshot Isolation*
}

\author{
Sameh Elnikety \\ School of Computer and \\ Communication Sciences \\ EPFL \\ Lausanne, Switzerland
}

\author{
Fernando Pedone \\ Faculty of Informatics \\ Università della Svizzera Italiana \\ USI \\ Lugano, Switzerland
}

\author{
Willy Zwaenepoel \\ School of Computer and \\ Communication Sciences \\ EPFL \\ Lausanne, Switzerland
}

\begin{abstract}
Generalized snapshot isolation extends snapshot isolation as used in Oracle and other databases in a manner suitable for replicated databases. While (conventional) snapshot isolation requires that transactions observe the "latest" snapshot of the database, generalized snapshot isolation allows the use of "older" snapshots, facilitating a replicated implementation. We show that many of the desirable properties of snapshot isolation remain. In particular, read-only transactions never block or abort and they do not cause update transactions to block or abort. Moreover, under certain assumptions on the transaction workload the execution is serializable.

An implementation of generalized snapshot isolation can choose which past snapshot it uses. An interesting choice for a replicated database is prefix-consistent snapshot isolation, in which the snapshot contains at least all the writes of locally committed transactions. We present two implementations of prefix-consistent snapshot isolation. We conclude with an analytical performance model of one implementation, demonstrating the benefits, in particular reduced latency for read-only transactions, and showing that the potential downsides, in particular change in abort rate of update transactions, are limited.
\end{abstract}

\section{Introduction}

There is increasing interest in replicating the generation of dynamic Web content $[2,14]$ for many Web services to achieve efficiency and fault tolerance. The user's request is executed on a "nearby" replica, thereby avoiding long roundtrip delays and distributing the load over the replicas. In addition, replication increases the availability of a Web

${ }^{*}$ This research was partially supported by the Swiss National Science Foundation grant number 200021-107824: System Support for Distributed Dynamic Content Web Services. service because if a replica fails, information can still be accessed at other replicas. In a centralized dynamic content Web site, the dynamic data is most often stored in a database $[8,3]$. Therefore, one of the key problems in replicating dynamic Web content generation is replicating the database and keeping the replicas consistent.

Ideally, we would like to provide the same database consistency as in the centralized database case. We focus here on databases providing snapshot isolation [6]. In such a database, a transaction $T$ obtains at the beginning of its execution the latest snapshot of the database, reflecting the writes of all transactions that have committed before transaction $T$ starts. At commit, the database checks that the writeset of transaction $T$ does not intersect with the writesets of the transactions that committed since $T$ 's snapshot. If there is a non-zero intersection, transaction $T$ aborts; otherwise, it commits.

Snapshot isolation is popular for a number of reasons, one of which is the pragmatic reason that Oracle $[9,20]$ and other database vendors [25, 32, 19] use it. More fundamentally, snapshot isolation never requires read-only transactions to block or abort, and read-only transactions do not cause update transactions to block or abort. This advantage is significant for workloads with a large fraction of readonly transactions (such as those resulting from the generation of dynamic Web content). Snapshot isolation provides a weaker form of consistency than serializability, but one that programmers can understand and use. Moreover, Fekete et al. [11,13] have recently demonstrated that under certain conditions on the workload transactions executing on a database with snapshot isolation produce serializable histories.

\subsection{Generalized Snapshot Isolation (GSI)}

Extending snapshot isolation to replicated databases is not straightforward. Intuitively, the problem stems from the requirement that a transaction must see the "latest" snapshot when it starts execution. In contrast with the central- 
ized case, the notion of "latest" is not a priori well-defined in a distributed setting. Implementations of an ordering that defines the notion of "latest" and makes the corresponding snapshot available may impose a delay at the start of a transaction. Imposing such a delay at the beginning of read-only transactions voids one of the main benefits of snapshot isolation.

Generalized snapshot isolation is based on the observation that a transaction need not necessarily observe the "latest" snapshot. It can observe an older snapshot, and many properties as those in (conventional) snapshot isolation continue to hold. Conditions can be identified that guarantee serializable execution. With a suitable choice of "older", readonly transactions execute without delay or aborts, and they do not cause update transactions to block or abort. Transactions may, however, observe somewhat older data. To commit an update transaction, its writeset must be checked against the writesets of recently committed transactions, as before. The probability of an abort increases, as it becomes more likely that at the time of its commit an update transaction finds that another transaction has written to the same data as it did since the time of its snapshot.

The ability to start from an older snapshot gives rise to an interesting spectrum of possibilities with attendant performance tradeoffs. At one end of the spectrum is the conventional notion of snapshot isolation, further referred to in this paper as conventional snapshot isolation, in which each transaction reads the latest snapshot. This is clearly appropriate in a centralized setting where the latest snapshot is trivially available, and where using the latest snapshot minimizes the probability of aborts. At the other end of the spectrum is the trivial solution in which all transactions observe the initial snapshot (i.e., the database state as in the beginning of execution).

\subsection{Prefix-Consistent Snapshot Isolation (PCSI)}

In a replicated setting, an interesting positioning in this spectrum is for a transaction to take as its initial snapshot the latest snapshot that is locally available on its replica. We capture this idea in prefix-consistent snapshot isolation. As an instance of generalized snapshot isolation, prefixconsistent snapshot isolation maintains the desirable properties of generalized snapshot isolation. Read-only transactions never block or abort, and they do not cause update transactions to block or abort. Moreover, transactions observe at least all the writes that have committed on the local replica before they start. This property is important in a workflow of transactions, in which a user submits a sequence of transactions to a replica.

\subsection{Contributions}

The contributions of this paper are as follows: (1) introducing generalized snapshot isolation, (2) establishing two sufficient conditions (one dynamic condition and one statically checkable condition) that guarantee serializable execution under generalized snapshot isolation, (3) introducing prefix-consistent snapshot isolation and two implementation strategies for replicated databases, and (4) analytically characterizing the relative performance of prefix-consistent snapshot isolation to conventional snapshot isolation.

\subsection{Roadmap}

This paper is organized as follows: We present the database model in Section 2 and develop generalized snapshot isolation in Section 3. Then we discuss how to guarantee serializability in Section 4, and provide a dynamic and a static condition for serializability under generalized snapshot isolation.

In section 5, we present prefix-consistent snapshot isolation, which is an instance of generalized snapshot isolation that is suitable for replicated databases. In section 6 , we present a model for a distributed system with replicated databases. We first show that conventional snapshot isolation must impose a delay on read-only transactions in that model, and then we develop two algorithms that implement prefix-consistent snapshot isolation , one using centralized certification and the other using distributed certification. In Section 7, we compare analytically the performance of prefix-consistent snapshot isolation to conventional snapshot isolation. Finally, we discuss related work in Section 8 and derive our conclusions and future research directions in Section 9.

We omit the proofs of propositions and of correctness of the algorithms due to space limitations. We, however, provide all proofs in a technical report [10].

\section{Database Model and Definitions}

\subsection{Database Model}

We assume that a database is a collection of uniquely identified data items. Several versions of each data item may co-exist simultaneously in the database, but there is a total order among the versions of each data item. A snapshot of the database is a committed state of the database.

A transaction $T_{i}$ is a sequence of read and write operations on data items, followed by either a commit or an abort operation. We denote $T_{i}$ 's write operation on item $X$ by $W_{i}\left(X_{i}\right)$. If $T_{i}$ executes $W_{i}\left(X_{i}\right)$ and commits, then a new version of $X$, denoted by $X_{i}$, is added to the database. Moreover, we denote $T_{i}$ 's read operation on item $X_{j}$ by 
$R_{i}\left(X_{j}\right) . T_{i}$ 's commit or abort is denoted by $C_{i}$ or $A_{i}$, respectively. Finally, to simplify the presentation, we assume that transactions do not contain redundant read or write operations $^{1}$ : A transaction reads any item at most once and writes any item at most once, and if a transaction writes an item, it does not read that item afterwards.

A transaction is read-only if it contains no write operation, and is update otherwise. The readset of transaction $T_{i}$, denoted readset $\left(T_{i}\right)$, is the set of data items that $T_{i}$ reads. Similarly, the writeset of transaction $T_{i}$, denoted writeset $\left(T_{i}\right)$, is the set of data items that $T_{i}$ writes. We add additional information to the writesets to include the new values of the written data items.

A history $h$ over a set of transactions $\mathcal{T}=$ $\left\{T_{1}, T_{2}, \ldots, T_{n}\right\}$ is a partial order $\prec$ such that (a) $h$ contains the operations of each transaction in $\mathcal{T}$; (b) for each $T_{i} \in \mathcal{T}$, and all operations $O_{i}$ and $O_{i}^{\prime}$ in $T_{i}$ : if $O_{i}$ precedes $O_{i}^{\prime}$ in $T_{i}$, then $O_{i} \prec O_{i}^{\prime}$ in $h$; and (c) if $T_{i}$ reads $X$ from $T_{j}$, then $W_{j}\left(X_{j}\right) \prec R_{i}\left(X_{j}\right)$ in $h$ [7].

\subsection{Impacting Transactions}

To simplify some definitions in the paper, we assign a distinct time to each database operation, resulting in a total order consistent with the partial order $\prec$ for history $h$.

In generalized snapshot isolation, each transaction observes a snapshot of the database that is taken at some time, denoted snapshot $\left(T_{i}\right)$. If transaction $T_{i}$ sees a snapshot of the database taken at time snapshot $\left(T_{i}\right)$, then this snapshot includes the updates of all transactions that have committed before snapshot $\left(T_{i}\right)$. To argue about the timing relationships among transactions, we use the following definitions with respect to transaction $T_{i}$ :

- $\operatorname{snapshot}\left(T_{i}\right)$ : the time when $T_{i}$ 's snapshot is taken.

- $\operatorname{start}\left(T_{i}\right)$ : the time of the first operation of $T_{i}$.

- $\operatorname{commit}\left(T_{i}\right)$ : the time of $C_{i}$, if $T_{i}$ commits.

- $\operatorname{abort}\left(T_{i}\right)$ : the time of $A_{i}$, if $T_{i}$ aborts.

- $\operatorname{end}\left(T_{i}\right)$ : the time of either $C_{i}$ or $A_{i}$.

Notice that $\operatorname{snapshot}\left(T_{i}\right) \leq \operatorname{start}\left(T_{i}\right)<\operatorname{end}\left(T_{i}\right)$. The time of the first operation of $T_{i}$ defines $\operatorname{start}\left(T_{i}\right)$ since we do not use an explicit transaction begin operation. Next, we define the relation impacts for update transactions.

$$
\begin{aligned}
& \text { - } \frac{T_{j} \text { impacts } T_{i} \text { iff }}{\operatorname{writeset}\left(T_{i}\right)} \cap \operatorname{writeset}\left(T_{j}\right) \neq \emptyset \text {, and } \\
& \operatorname{snapshot}\left(T_{i}\right)<\operatorname{commit}\left(T_{j}\right)<\operatorname{commit}\left(T_{i}\right)
\end{aligned}
$$

\footnotetext{
${ }^{1}$ This assumption is not restrictive: These redundant operations can be added to the model, but they will complicate the presentation (e.g., the rules D1 and D2 of GSI). Moreover, in practice if a transaction contains redundant operations, then they can be removed using local variables in the program that issues the transaction [22].
}

From this definition, only committed update transactions may impact update transaction $T_{i}$. Read-only transactions and uncommitted transactions cannot impact $T_{i}$. When committing an active update transaction $T_{i}$, we say " $T_{j}$ impacts $T_{i}$ " to mean that if $T_{i}$ were to commit now, then $T_{j}$ would impact $T_{i}$.

\section{Generalized Snapshot Isolation (GSI)}

Generalized snapshot isolation (GSI) has two rules: the first regulates read operations, and the second regulates commit operations. For any history $h$ created by GSI, the following two properties hold: (The definitions below implicitly assume that indexes $i, j$, and $k$ are different values.)

- D1. (GSI Read Rule)

$$
\begin{aligned}
& \forall T_{i}, X_{j} \text { such that } R_{i}\left(X_{j}\right) \in h: \\
& \text { 1- } W_{j}\left(X_{j}\right) \in h \text { and } C_{j} \in h ; \\
& \text { 2- } \operatorname{commit}\left(T_{j}\right)<\operatorname{snapshot}\left(T_{i}\right) ; \\
& \text { 3- } \forall T_{k} \operatorname{such} \text { that } W_{k}\left(X_{k}\right), C_{k} \in h: \\
& \quad\left[\operatorname{commit}\left(T_{k}\right)<\operatorname{commit}\left(T_{j}\right)\right. \text { or } \\
& \left.\quad \operatorname{snapshot}\left(T_{i}\right)<\operatorname{commit}\left(T_{k}\right)\right] .
\end{aligned}
$$

- D2. (GSI Commit Rule)

$$
\frac{\forall T_{i}, T_{j} \text { such that } C_{i}, C_{j} \in h:}{\text { 4- } \neg\left(T_{j} \text { impacts } T_{i}\right) .}
$$

The read rule D1 ensures that each transaction reads only committed data, that is, each transaction observes a committed snapshot of the database. This snapshot could be any snapshot that has been taken before the transaction starts, which can be provided efficiently in a distributed system.

Part one and two of D1 allow $T_{i}$ to read the data items written by transactions that committed before $T_{i}$ 's snapshot. The third part of D1 prevents holes in the snapshot that $T_{i}$ receives: If $T_{i}$ sees a snapshot at time $t$ and reads data item $X$, then it reads $X$ from the last committed transaction at time $t$ that updated $X$.

The commit rule D2 prevents any update transaction $T_{i}$ from committing if it is impacted by another committed update transaction. From the definition of the relation impacts, D2 is trivially satisfied for read-only transactions. This rule gives the "first-committer-wins behavior" [7]: If two active transactions update the same item, then the transaction that commits first succeeds at updating that item, while the other transaction aborts.

When an update transaction $T_{i}$ requests to commit in a database running GSI, the database performs a test to decide if $T_{i}$ can commit or must abort. This test is called certification. In other words, to dynamically enforce the commit rule D2 when deciding whether an update transaction $T_{i}$ can commit, the database certifies $T_{i}$ : the database checks whether writeset $\left(T_{i}\right)$ intersects with the writeset of any update transaction that committed after $\operatorname{snapshot}\left(T_{i}\right)$. If all intersections are empty, $T_{i}$ commits; otherwise $T_{i}$ aborts. 
Conventional snapshot isolation (CSI) is a special case of GSI where each transaction sees the last snapshot with respect to its starting time, i.e., $\operatorname{snapshot}\left(T_{i}\right)=\operatorname{start}\left(T_{i}\right)$. This is referred to in literature simply as "snapshot isolation". On a system composed of a single site, it is easy to implement conventional snapshot isolation, as the latest snapshot is available for use without delay. Using the latest snapshot, in general, minimizes the number of transactions that may impact an update transaction. Therefore, in a centralized database CSI typically reduces the abort rate of update transactions and provides transactions with the most recent snapshots.

\section{Serializability under GSI}

We now turn to discussing serializability under GSI. Serializability is important: it is the basic correctness criteria in much work on databases [7, 22]. If applications need serializability, then providing a weaker form of consistency makes those applications much harder to develop. GSI provides a weaker form of consistency than serializability.

Both GSI and CSI may produce non-serializable histories. Here is an example of the write-skew anomaly [6]. Assume that $X$ and $Y$ are the balances of two bank accounts for the same person and that the bank enforces the constraint $(X+Y) \geq 0$. The next two procedures deduct an input amount, $A 1$ and $A 2$, from $X$ and $Y$, respectively.

$$
\begin{aligned}
& \operatorname{Proc}_{i}=\{\text { input } A 1 ; \operatorname{read} X \text { and } Y ; \\
& \text { if }((X+Y)<A 1) \text { then } \text { abort\} } \\
& \text { else }\{X=X-A 1 \text {; write } X \text {; commit }\} \text { \} } \\
& \text { Proc }_{j}=\{\text { input } A 2 ; \operatorname{read} X \text { and } Y \text {; } \\
& \text { if }((X+Y)<A 2) \text { then } \text { \{abort } \\
& \text { else }\{Y=Y-A 2 \text {; write } Y \text {; commit }\} \quad\}
\end{aligned}
$$

Running these procedures concurrently as transactions $T_{i}$ and $T_{j}$ can produce the following non-serializable history under CSI. Assume that $X_{0}, Y_{0}, A 1$, and $A 2$ are 50, 50, 60, and 60, respectively. $h_{1}=R_{i}\left(X_{0}=50\right), R_{i}\left(Y_{0}=\right.$ $50), R_{j}\left(X_{0}=50\right), R_{j}\left(Y_{0}=50\right), W_{i}\left(X_{i}=-10\right), C_{i}$, $W_{j}\left(Y_{j}=-10\right), C_{j}$.

\subsection{Dynamic Serializability Condition (D3)}

D3 is a dynamic condition: it adds a new rule to GSI. Transactions that satisfy condition D3 produce serializable histories under GSI.

- D3. (Dynamic Serializability Rule)

$$
\frac{\forall \text { update transactions } T_{i}, T_{j} \text { such that } C_{i}, C_{j} \in h \text { and }}{\frac{\operatorname{snapshot}\left(T_{i}\right)<\operatorname{commit}\left(T_{j}\right)<\operatorname{commit}\left(T_{i}\right):}{\operatorname{readset}\left(T_{i}\right) \cap \operatorname{writeset}\left(T_{j}\right)=\emptyset .}}
$$

PROPOSITION 1. GSI is serializable under D3.

To use this condition we add rule D3 to the two rules, D1 and D2, of GSI. Thus, rules D1, D2, D3 are applied at runtime. First when certifying an update transaction $T_{i}$, GSI requires $T_{i}$ not to have any impacting transaction, which can be verified by comparing writeset $\left(T_{i}\right)$ to the writesets of transactions that committed since $T_{i}$ 's snapshot, i.e., $\left\{T_{j}: \operatorname{snapshot}\left(T_{i}\right)<\operatorname{commit}\left(T_{j}\right)\right\}$. Second, condition D3 requires readset $\left(T_{i}\right)$ not to intersect with the writesets of the same set of committed update transactions $\left\{T_{j}: \operatorname{snapshot}\left(T_{i}\right)<\operatorname{commit}\left(T_{j}\right)\right\}$. Therefore, to certify $T_{i}$ both its readset and writeset must be available for the dynamic checks, as well as the writesets of the update transactions which committed since $T_{i}$ 's snapshot.

D3 is sufficient but not necessary. Consider this history: $h_{2}=W_{i}\left(X_{i}\right), C_{i}, W_{j}\left(X_{j}\right), R_{k}\left(X_{i}\right), W_{k}\left(Y_{k}\right), C_{j}, C_{k}$. History $h_{2}$ is serializable but does not satisfy D3.

Using the readsets is likely to incur a higher overhead than using only writesets, especially in a distributed setting. Next we present static condition S1, which avoids requiring the readset to certify an update transaction. Roughly speaking, "if we can determine the readsets and writesets of transactions statically, then we can apply condition D3 on all transaction pairs statically rather than dynamically". S1 allows only a subset of the histories allowed by D3.

\subsection{Static Serializability Condition (S1)}

$\mathrm{S} 1$ is a static condition; it adds no extra dynamic check to GSI. This condition can be checked statically by examining the mix of transactions and query templates in application programs. It can also be enforced by changing the transactions as described later.

- S1. (Static Serializability Condition)

$\forall$ update transactions $T_{i}, T_{j}$ :

$$
\left[\begin{array}{c}
\operatorname{writeset}\left(T_{i}\right) \cap \operatorname{writeset}\left(T_{j}\right) \neq \emptyset \\
\vee \\
\left(\begin{array}{c}
\operatorname{readset}\left(T_{i}\right) \cap \operatorname{writeset}\left(T_{j}\right)=\emptyset \\
\wedge \\
\operatorname{writeset}\left(T_{i}\right) \cap \operatorname{readset}\left(T_{j}\right)=\emptyset .
\end{array}\right)
\end{array}\right]
$$

PROPOSITION 2. GSI is serializable under SI.

$\mathrm{S} 1$ is sufficient but not necessary. For example, consider this history: $h_{3}=W_{i}\left(X_{i}\right), C_{i}, W_{j}\left(X_{j}\right), R_{k}\left(X_{i}\right), W_{k}\left(Y_{k}\right)$, $C_{k}, C_{j}$. History $h_{3}$ does not satisfy $\mathrm{S} 1$ because write$\operatorname{set}\left(T_{j}\right) \cap$ readset $\left(T_{k}\right) \neq \emptyset$. However, $h_{3}$ is serializable and is allowed by condition D3.

Read-only transactions trivially satisfy condition S1. Therefore in practice, to use condition S1 all possible update transactions must be known. That is usually the case in many database applications where database transactions are 
hidden from clients who interact with the system through a standard interface (e.g., application forms which contain SQL templates). In such a system, ad-hoc update transactions must be prevented, unless they are checked to satisfy condition S1. Fortunately, in many situations such ad-hoc update transactions are forbidden because they need to follow the procedures of the business logic. Nonetheless, adhoc read-only transactions are allowed because they satisfy S1 trivially.

To check if an application satisfies condition S1, we need to conservatively check every pair of update transactions. Some optimizations are possible if application specific information are available. For example, we do not need to check pairs of transactions that never run concurrently (i.e., are not active at the same time) in the database application. Such cases happen when two transactions always run sequentially in a single workflow as explained in Section 5, or when one transaction runs during business hours and the other runs at night.

If a pair of two update transactions do not satisfy $\mathrm{S} 1$, it must be that they do not write any data item in common and one of them writes a data item, $X$, and the other reads that data item. We can change the second transaction, which reads $X$, to include an identity write on item $X$, i.e., change $R(X)$ into $R(X), W(X)$. This change makes their writesets intersect; therefore, they now satisfy $\mathrm{S} 1$.

Checking update transactions-and altering them if needed - can be automated [11, 13]. Moreover, in some applications all transactions already satisfy $\mathrm{S} 1$. For example, the TPC-C and TPC-W benchmarks [30] are the industrystandard for evaluating online transaction processing systems and e-commerce systems, respectively. We, as well as other researchers [13], have verified that both benchmarks satisfy condition $\mathrm{S} 1$ by examining all update transactions from the specification documents: TPC-C and TPC-W produce serializable histories under GSI without any modification.

\section{Prefix-consistent Snapshot Isolation (PCSI)}

GSI's rules D1 and D2 do not specify which snapshot of the database a transaction should observe. The range of possible snapshots that a transaction can receive varies from the initial database state until the latest snapshot as in CSI.

Although applications can in general trade snapshot freshness for performance, especially in distributed environments, in some cases a transaction should be guaranteed to receive a snapshot that contains the updates of specific previous transactions. This happens, for example, when a transaction may need to observe the effects of previous transactions in the same application workflow. In order to capture this additional requirement on database snapshots, we introduce prefix-consistent snapshot isolation (PCSI).
We define PCSI in this section, and in the next section we present a model for replicated databases and instantiate the workflow relation and give an example to show its importance. Then we discuss how to implement PCSI in replicated databases.

PCSI is parameterized by an equivalence relation $\sim$ among transactions. If transactions $T_{i}$ and $T_{j}$ are in the same workflow, denoted by $T_{i} \sim T_{j}$, and $T_{j}$ commits before $T_{i}$ starts, then $T_{i}$ 's snapshot must be recent enough to contain at least $T_{j}$ 's updates, that is, if $T_{i} \sim$ $T_{j}$ and $\operatorname{commit}\left(T_{j}\right)<\operatorname{start}\left(T_{i}\right)$ then $\operatorname{commit}\left(T_{j}\right)<$ snapshot $\left(T_{i}\right)$. We add a new conjunction, part 4 below, to GSI rules D1 and D2 to obtain the following two properties which must hold for any history generated by PCSI.

- P1. (PCSI Read Rule)

$$
\begin{aligned}
& \forall T_{i}, X_{j} \text { such that } R_{i}\left(X_{j}\right) \in h: \\
& \hline \text { 1- } W_{j}\left(X_{j}\right) \in h \text { and } C_{j} \in h ; \\
& \text { 2- } \operatorname{commit}\left(T_{j}\right)<\operatorname{snapshot}\left(T_{i}\right) ; \\
& \text { 3- } \forall T_{k} \operatorname{such} \text { that } W_{k}\left(X_{k}\right), C_{k} \in h: \\
& \quad\left[\operatorname{commit}\left(T_{k}\right)<\operatorname{commit}\left(T_{j}\right)\right. \text { or } \\
& \left.\quad \operatorname{snapshot}\left(T_{i}\right)<\operatorname{commit}\left(T_{k}\right)\right] ; \\
& \text { 4- } \forall T_{k} \operatorname{such} \text { that } W_{k}\left(X_{k}\right), C_{k} \in h \text { and } T_{i} \sim T_{k}: \\
& {\left[\operatorname{commit}\left(T_{k}\right)<\operatorname{commit}\left(T_{j}\right)\right. \text { or }} \\
& \left.\operatorname{start}\left(T_{i}\right)<\operatorname{commit}\left(T_{k}\right)\right] .
\end{aligned}
$$

- P2. (PCSI Commit Rule)

$$
\frac{\forall T_{i}, T_{j} \text { such that } C_{i}, C_{j} \in h:}{\text { 5- } \neg\left(T_{j} \text { impacts } T_{i}\right) .}
$$

Since GSI does not allow holes in the snapshot, it follows that $T_{i}$ 's snapshot includes the effects of all transactions that are in $T_{i}$,s workflow and that have committed before $T_{i}$ starts. This does not imply that transactions executing under PCSI will see only up-to-date snapshots of the database as in CSI (and pay the corresponding implementation cost): $T_{i}$ 's snapshot may not include the updates of some transactions that have committed before $T_{i}$ starts and that are not in $T_{i}$ 's workflow.

\section{Replicated databases}

In this section we present a model for a distributed system with replicated databases. We show that read-only transactions under CSI must block in this model. Then, we instantiate PCSI's workflow relation and discuss two algorithms that implement PCSI. Both algorithms implement only PCSI; if serializability is needed, then condition D3 or S1 should be applied.

\subsection{System Model and Definitions}

We consider an asynchronous distributed system composed of a set of database sites $S_{1}, S_{2}, \ldots, S_{i}, \ldots$, which 


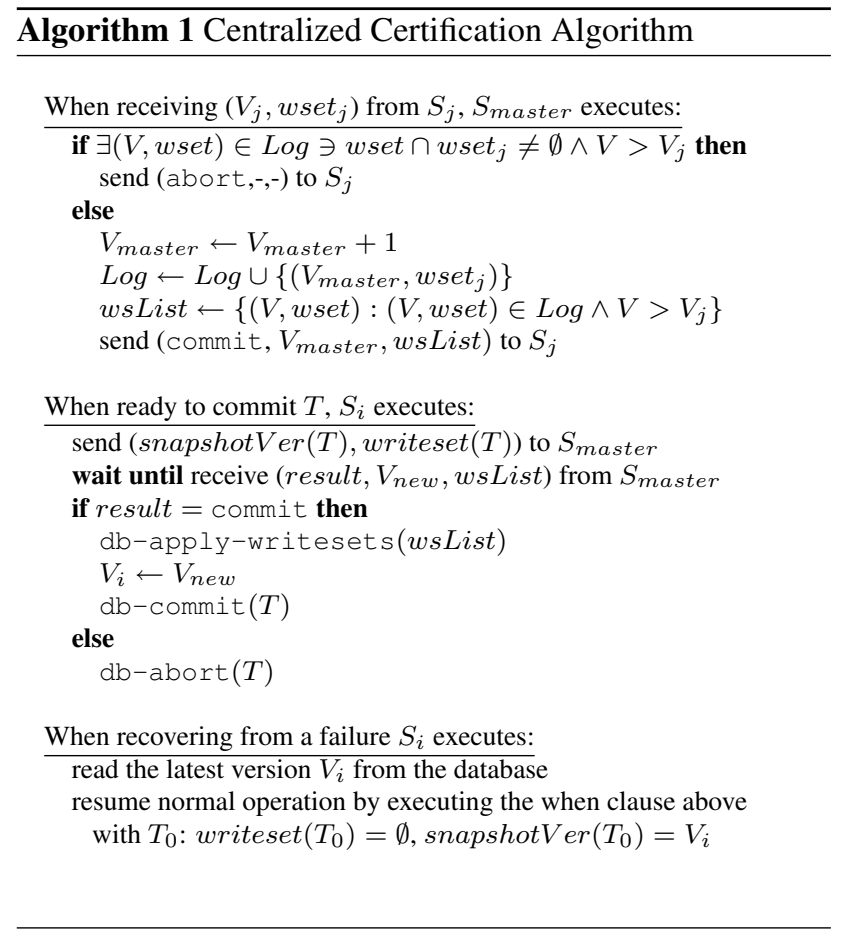

communicate through reliable message passing. We make no assumptions about the time it takes for sites to execute and for messages to be transmitted. Each database site has a full copy of the database. A transaction is (globally) committed as soon as it is locally committed at any site.

CSI requires each transaction to observe the latest snapshot. Under CSI in a replicated setting, when a database site receives a transaction, it has to provide that transaction with the latest snapshot. However, doing so may force the site either to delay the start of the transaction to obtain the latest snapshot, or to stop accepting new transactions during some time intervals.

As stated in Proposition 3, in this model any implementation of CSI requires transactions to block. By definition, in a blocking implementation, there are periods of time during which sites cannot start new transactions. In a nonblocking implementation, transactions can start at any site at any time. Blocking voids the main benefit of CSI and motivates using PCSI.

PROPOSITION 3. There is no non-blocking implementation of CSI in an asynchronous system, even if database sites never fail.

Although Proposition 3 holds in the absence of failures, ${ }^{2}$ we assume the more general model, crash-recovery,

\footnotetext{
${ }^{2}$ If transactions are allowed to block and database sites do not fail, then there exists a simple algorithm which implements CSI in an asynchronous system [10]
}

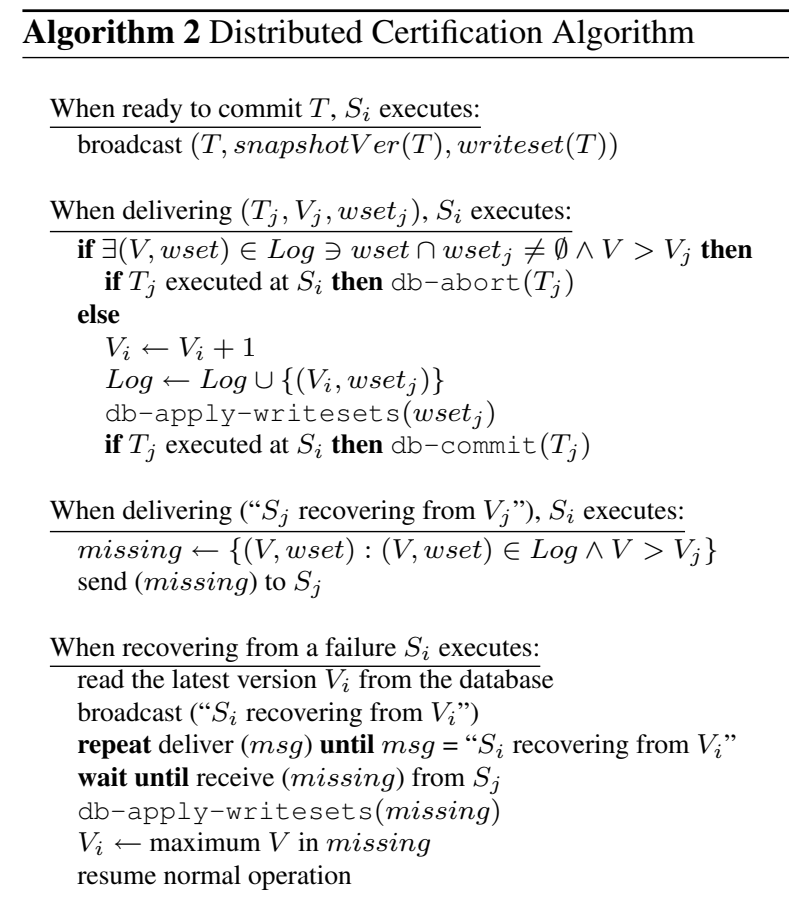

where sites may fail independently by crashing, and subsequently recover an unbounded number of times. We do not consider Byzantine failures.

We present two algorithms that implement PCSI. The first algorithm uses a central certifier to certify update transactions. The central certifier is a single point of failure, and this motivates the second algorithm which replicates the certifier using the state-machine approach [27]. The second algorithm employs an atomic broadcast abstraction [18] that is defined by the primitives broadcast $(m)$ and deliver $(m)$, which satisfy these properties: if a site delivers a message $m$, then all sites eventually deliver $m$ (agreement); if two sites, $S_{i}$ and $S_{j}$, both deliver messages $m_{1}$ and $m_{2}$, then they do so in the same order (total order); if a site broadcasts a message and does not crash, then all sites deliver the message (validity); and a message is only delivered if it was previously broadcast by some site (integrity).

In both algorithms, every transaction executes its database operations at a single site-except for the commit operation of an update transaction as it requires remote communication to certify the transaction. Upon the commit of an update transaction, its writeset is applied at the other replicas, according to the algorithms.

We define the workflow relation $\sim$ as $\left\{\left(T_{i}, T_{j}\right): T_{i}\right.$ and $T_{j}$ execute in the same database site $\}$. To illustrate the need for the workflow relation consider the following scenario: A client first issues (and successfully commits) a transaction to database site $S_{i}$ requesting to buy a book. Then, she 
issues a second transaction to the same site requesting a list of her orders. The above instantiation of the workflow relation guarantees that the second transaction observes the effects of the first transaction; the list of orders contains the order for that book.

We use version numbers to approximate global time. The database goes through a number of versions, each identified by a monotonically increasing version number. When a transaction starts, it obtains one of these versions as its initial snapshot. We use the notation snapshot $\operatorname{er}\left(T_{i}\right)$ for the version number of the initial snapshot of transaction $T_{i}$ and commitVer $\left(T_{i}\right)$ for the version number of the database produced after the commit of transaction $T_{i}$. These two version numbers correspond to the global times snapshot $\left(T_{i}\right)$ and $\operatorname{commit}\left(T_{i}\right)$, respectively.

\subsection{Centralized Certification Algorithm}

One site is the master (or central certifier). The master certifies the commits of update transactions, and it stores the writesets of all committed update transactions. Replicas communicate only with the master using reliable message passing and do not communicate among each other.

The master maintains a persistent $\log$ which is a set of (version number, writeset) tuples; Log is not lost in the event of a crash . Each tuple in Log contains the writeset of the transaction that produced the database version with that particular version number. Also, the master maintains the current version number $V_{\text {master }}$, initially set to zero.

Each replica $S_{i}$ maintains its own version number $V_{i}$, which indicates the version number of its current database version, and which may be different from the current version at the master or at other replicas. For each transaction $T$ that is active at a replica, the replica maintains the version number of its starting snapshot, which is denoted snapshot $\operatorname{Ver}(T)$.

At the start of transaction $T, S_{i}$ provides the transaction with a snapshot equal to its current database version, and assigns $V_{i}$ to snapshot $\operatorname{er}(T)$. Reads and writes execute locally against this snapshot, without any communication. When a read-only transaction completes, nothing further needs to be done. When an update transaction completes, it needs to be certified to commit successfully, as described in Algorithm 1.

When a database site recovers from a failure, it reads its last committed snapshot from the disk. Then, it sends the version of that snapshot to the certifier to obtain the most recent updates.

The master can garbage collect the Log data structure by deleting old entries. To allow a replica to recover by exchanging information only with the master and without communicating with other replicas, the versions older than the minimum $V_{i}$ for all replicas can be safely deleted from the $\log$. When certifying a transaction, if any writeset needed to certify that transaction has been deleted from $\log$, the transaction is aborted to preserve the safety properties ensured by certification. This is a trade-off between the abort rate and the size of persistent storage.

\subsection{Distributed Certification Algorithm}

The distributed algorithm is a natural extension of the centralized one, using the state machine approach [27, 23]. Each replica both executes transactions and certifies update transactions. The algorithm uses an atomic broadcast abstraction [18] to deliver the writesets for certification to all replicas.

The key difference from the centralized implementation is that all replicas execute certification. Thus, all replicas now maintain the data structures that were maintained by the master in the centralized implementation. In particular, the $\log$ data structure is persistent at each database site.

Transaction operations, except for the commits of update transactions, are handled as in the centralized algorithm.

After executing an update transaction $T$, the replica broadcasts a certification request, containing snapshot $\operatorname{Ver}(T)$ and writeset $(T)$ to all replicas. As depicted in Algorithm 2, when replica $S_{i}$ delivers the certification request, it applies the commit rule P2 and checks that no committed transaction impacts $T$. If so, $T$ is aborted; otherwise, $T$ is committed.

When a database site crashes and later recovers, it needs to apply the effects of all messages which it did not deliver or which it delivered but did not process due to the crash. On recovery, the database site reads its latest snapshot from the database to determine $V_{i}$. Then it broadcasts a recovery message. The replica ignores all messages it delivers until it delivers the recovery message. Then the replica waits for a message with the updates it missed while it was down, applies them to the database, and continues processing delivered messages as usual.

\section{Performance Analysis of PCSI}

This section assesses analytically the relative performance of PCSI to CSI. More specifically, we show that under certain assumptions the transaction abort rate is a linear function of both transaction length and the age of the snapshot that the transaction observes.

We use a simple model to estimate the abort rate of update transactions. This model is used to predict the probability of waits and deadlocks in centralized [17, pp. 428] and replicated databases [16]. We assume that the abort rate is small. This is a self-regulating assumption: If the abort rate is too high, then snapshot isolation algorithms are not suitable for the workload. 


\begin{tabular}{||r|l||}
\hline Symbol & Meaning \\
\hline$C W$ & length of the conflict window \\
\hline$C W\left(T_{i}\right)$ & conflict window of transaction $T_{i}$, which is $\left[\operatorname{snapshot}\left(T_{i}\right), \operatorname{end}\left(T_{i}\right)\right]$ \\
\hline$D$ & age of the snapshot that the transaction receives when it begins execution, which is $\operatorname{start}\left(T_{i}\right)-\operatorname{snapshot}\left(T_{i}\right)$ \\
\hline$D B S i z e$ & database size (total number of data items) \\
\hline$L$ & number of seconds needed to execute a transaction on a single database \\
\hline$N$ & number of database sites \\
\hline$R R$ & $\begin{array}{l}\text { request-reply delay: delay for replica to send message to master and receive response, including round-trip, data } \\
\text { transfer and message processing }\end{array}$ \\
\hline$T P S$ & number of transactions per second \\
\hline$W$ & number of data items updated by each transaction \\
\hline
\end{tabular}

Table 1. Parameters of the analytical model.

Initially, we consider a single-site database to contrast GSI to CSI. Then, we consider a replicated database over multiple sites to compare the abort rate of PCSI to that of CSI using an implementation based on centralized certification. Finally, using that implementation we compare the response times of read-only and update transactions in both PCSI and CSI.

\subsection{Model}

We consider a single database site that uses GSI under the following assumptions. The database has a fixed set of data items. The total number of these data items is DBSize. Here we consider only update transactions to compute their abort rate; read-only transactions are ignored. The database originates $T P S_{\text {update }}$ update transactions per second. Each transaction updates $W$ data items, takes $L$ seconds to finish, and observes a snapshot of the database that is $D$ seconds old. Table 1 lists the model parameters.

We define the time interval $C W\left(T_{i}\right)$, the conflict window of transaction $T_{i}$, such that $C W\left(T_{i}\right)=\left[\operatorname{snapshot}\left(T_{i}\right)\right.$, $\left.\operatorname{end}\left(T_{i}\right)\right]$. The length of the conflict window is denoted simply as $C W=(L+D)$. According to the commit rule D2, while certifying $T_{i}$ any transaction $T_{j}$ will force $T_{i}$ to abort if commit $\left(T_{j}\right) \in C W\left(T_{i}\right)$ and $T_{j}$ wrote a data item that is also written by $T_{i}$.

In this model, we assume that access to data items is uniform (no hotspots) and that DBSize $>>\left(T P S_{\text {update }} *\right.$ $L * W)$, which means that at any instant the number of data items updated is much smaller than the database size.

\subsection{Centralized Database Abort Rate}

In this subsection, we consider a centralized database using GSI to compute the abort rate of update transactions. The database artificially gives each update transaction a snapshot that is $D$ seconds old, even though the most recent snapshot is available. First we compute the probability that a single transaction, $T_{i}$, has to abort. On average, the number of transactions that commit in $C W\left(T_{i}\right)$, the conflict window of $T_{i}, \quad=\left(T P S_{\text {update }} * C W\right)$. Hence, the number of writes in that window $=W *\left(T P S_{\text {update }} * C W\right)$. The probability that a specific update (i.e., a write operation in $T_{i}$ ) conflicts with one of the writes in $C W\left(T_{i}\right)=$ (number of writes) $/$ (database size $)=\left(W * T P S_{\text {update }} *\right.$ $C W) / D B$ size. If any such conflict occurs, transaction $T_{i}$ must abort according to the commit rule D2. Since $T_{i}$ has $W$ of these updates, the probability that $T_{i}$ has to abort $=W *($ probability of a single conflict $)=\left(W^{2} *\right.$ $\left.T P S_{\text {update }} * C W\right) / D B$ Size.

Then we compute the transaction abort rate at the database site. The rate of aborted transactions $=$ (rate of transactions) * (probability that one transaction must abort) $=T P S_{\text {update }} *\left(W^{2} * C W * T P S_{\text {update }}\right) / D B$ Size. Therefore, the abort rate at the database site $=\left(T P S_{\text {update }}^{2} *\right.$ $\left.W^{2} / D B s i z e\right) *(C W)$. This rate is directly proportional to the length of the conflict window $(C W=\mathrm{D}+\mathrm{L})$.

Under CSI each transaction observes the latest snapshot of the database. Hence, $D=0$ and $C W=L$. The node abort rate $=\left(T P S_{\text {update }}^{2} * W^{2} / D B\right.$ size $) *(L)$.

For a single-site database the relative increase in node abort rate due to using GSI instead of CSI $=(1+(D / L))$, which depends on $D$, the snapshot age: the older the snapshot, the higher the relative increase in abort rate.

\subsection{Replicated Database Abort Rate}

We now turn to replicated databases and estimate the abort rate of update transactions under PCSI and CSI. We consider a database replicated over $N$ sites, where each site has a full database replica and originates $T P S_{\text {update }}$ update transactions per second. We use a simple implementation based on a master database to perform centralized certification for both PCSI and CSI. Hence, our performance evaluation is valid only for this implementation. The request-reply delay, $R R$, is the time necessary for a replica to send a message to the master and receive the response. $R R$ includes 


\begin{tabular}{|c|c|c|c|}
\hline Metric & prefix-consistent snapshot isolation & conventional snapshot isolation & ratio of PCSI/CSI \\
\hline $\begin{array}{l}\text { System abort } \\
\text { rate of update } \\
\text { transactions }\end{array}$ & $\begin{array}{l}\left(\left(N * T P S_{\text {update }} * W\right)^{2} / D \text { Bsize }\right) * \\
(D+L+0.5 * R R), \\
127 \text { trans/sec }(\% 1 \text { of update trans. })\end{array}$ & $\begin{array}{l}\left(\left(N * T P S_{\text {update }} * W\right)^{2} / D \text { Bsize }\right) * \\
(L+R R), \\
58 \text { trans/sec }(\% 0.5 \text { of update trans. })\end{array}$ & $\begin{array}{l}(D+L+0.5 * R R) / \\
(L+R R), \\
2.2\end{array}$ \\
\hline $\begin{array}{r}\text { Response time } \\
\text { of update } \\
\text { transactions }\end{array}$ & $\begin{array}{l}(L+R R) \\
250 \mathrm{~ms}\end{array}$ & $\begin{array}{l}(L+2 * R R) \\
450 \mathrm{~ms}\end{array}$ & $\begin{array}{l}(L+R R) / \\
(L+2 * R R) \\
0.55\end{array}$ \\
\hline $\begin{array}{r}\text { Response time } \\
\text { of read-only } \\
\text { transactions }\end{array}$ & $\begin{array}{l}(L) \\
50 \mathrm{~ms}\end{array}$ & $\begin{array}{l}(L+R R) \\
250 \mathrm{~ms}\end{array}$ & $\begin{array}{l}(L) /(L+R R), \\
0.2\end{array}$ \\
\hline
\end{tabular}

Table 2. Summary of the performance metrics, with values: $D=\mathbf{4 0 0} \mathrm{ms}, D B$ Size $=\mathbf{1 0}, 000,000, L=$ $50 \mathrm{~ms}, N=\mathbf{8}$ nodes, $R R=\mathbf{2 0 0} \mathrm{ms}, T P S=\mathbf{1 0 , 0 0 0}$ trans. $/ \mathrm{sec}$ (update trans. ratio $=\% 15, T P S_{\text {update }}=$ 1500), $W=4$.

the network round-trip delay, time necessary to transfer the data, and message processing delay. In wide-area networks, the round-trip delay constitutes a major part of $R R$.

From the previous subsection, the system abort rate is approximately $=\left(\left(N * T P S_{\text {update }}\right)^{2} * W^{2} / D B\right.$ size $) *(C W)$. For the replicated case, the abort rate rises rapidly as the number of replicas increases because the abort rate is a quadratic function of $N$. It remains to estimate the length of the conflict window $C W$.

Under PCSI each update transaction observes the most recent snapshot available locally at the replica without any delay as depicted in Figure 1. At commit time, the database replica sends the transaction writeset for certification to the master database in order to check that there is no impacting transaction. The length of the conflict window is $C W=$ $(D+L+0.5 * R R)$.

Under CSI each update transaction must observe the latest snapshot in the system as it arrives at a database site. We assume that this can be done by sending a message to the master database and by receiving the reply as in Figure 2. This reply either indicates that the site already has the latest snapshot, or includes the missing updates, which the database installs to obtain the latest snapshot. At commit, the replica sends the writeset of the new transaction to the master database for certification to ensure that it is not impacted by any transaction in the system. The length of the conflict window is $C W=(0.5 * R R+L+0.5 * R R)$. The relative increase in node abort rate due to using PCSI instead of CSI $=(D+L+0.5 * R R) /(L+R R)$.

\subsection{Replicated Database Response Time}

Here we consider both read-only and update transactions and estimate their response times. We define the response time of a transaction to be the time taken from receiving the transaction at a database site until knowing the commit or abort status at the same database site. For PCSI in Figure 1, the response times of update and read-only transactions are $(L+R R)$ and $(L)$, respectively. Similarly for CSI in Figure 2 , each transaction has to incur a round-trip delay to obtain the latest snapshot before it begins execution. The response times of update and read-only transactions are $(L+2 * R R)$ and $(L+R R)$, respectively.

The response times of PCSI are lower, at the cost of (1) letting transactions observe less recent data and (2) having a potentially higher abort rate for update transactions.

\subsection{Numerical Evaluation}

To get numeric estimates, we use "typical" numbers from a recent TPC-W disclosure report [29] and a requestresponse delay of $200 \mathrm{msec}$ [5]. We use these numbers because we intend to use PCSI in a wide-area replicated database and TPC-W is the industry standard benchmark for e-commerce systems $[8,30]$. We, however, vary the ratio between some of these parameters in Figures 3 and 4.

Table 2 summarizes our findings. In that particular environment, the abort rate of update transactions in both PCSI and CSI is small, and PCSI's abort rate is twice CSI's abort rate. In addition, the response time of update transactions in PCSI is half of the response time of update transactions in CSI. The response time of read-only transactions in PCSI is one fifth of CSI's response time of read-only transactions.

Figure 3 shows the ratio between the abort rate of update transactions in PCSI and CSI. The abort rate ratio is the rate of aborts in PCSI divided by the rate of aborts in CSI. The graph contains several curves corresponding to different values of D/L. For small values of RR/L, PCSI aborts more update transactions than CSI. Then as the ratio of RR/L increases, the trend reverses and CSI starts to abort more update transactions than PCSI. The ratio of abort rates reaches 0.5 for all curves as $\mathrm{RR} / \mathrm{L}$ gets very large, that is, 
(a) Prefix-Consistent Snapshot Isolation,

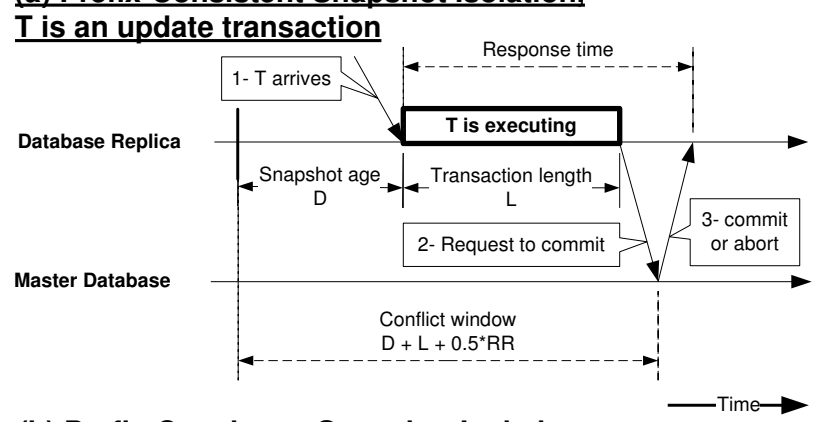

(b) Prefix-Consistent Snapshot Isolation.

$\underline{T}$ is a read-only transaction

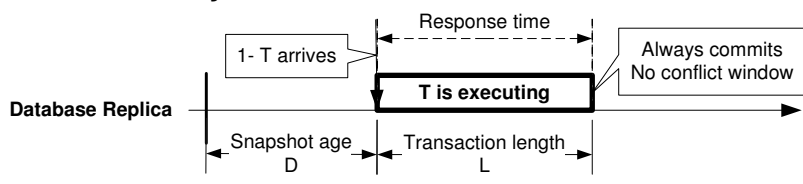

Master Database

Figure 1. Transaction execution in replicated database using PCSI with master database \& centralized certification.

PCSI aborts fewer update transactions than CSI. Although it is contrary to intuition for PCSI to abort fewer update transactions than CSI, it can be explained by the fact that when the response-request delay is large, waiting to get the latest snapshot increases the conflict window. This increase leads to the higher abort ratio of CSI compared to PCSI.

Figure 4 shows the ratio of response times of different transaction classes in PCSI to those in CSI. The response time ratio of a class of transactions is the average response time of that class of transactions in PCSI divided by the average response time of the same class in CSI. There are three curves corresponding to read-only transactions, update transactions, and "all transactions" which corresponds to the ratio between average response times of all transactions in PCSI and CSI. Both PCSI and CSI give the same response times when $R R=0$ (centralized environment); however, as RR/L increases PCSI gives substantially better response times for all transaction types. The response time ratio for update transactions approaches 0.5 quickly as RR/L increases.

This improvement in response times comes at a cost; transactions may not observe the latest snapshot in PCSI. The request-reply delay, $R R$, is constrained by the speed of light and $\mathrm{L}$ is reduced with faster computers, so RR/L increases as technology advances, favoring PCSI to CSI in both abort rates and response times. (a) Conventional Snapshot Isolation,

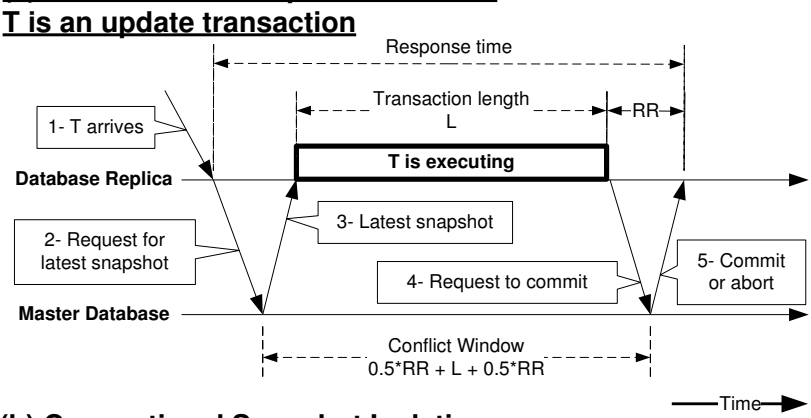

(b) Conventional Snapshot Isolation. $T$ is a read-only transaction

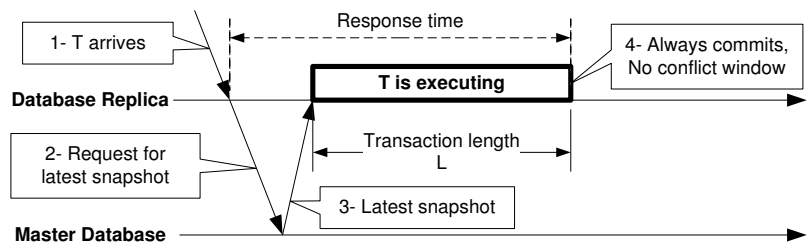
Figure 2. Transaction execution in repli-
cated database using CSI with master
database \& centralized certification.

\subsection{Summary}

For centralized databases, the abort rate under GSI is higher than that of CSI in which read-only transactions observe the latest snapshot. This makes CSI a clear winner in centralized systems.

For replicated databases, there is a tradeoff. Under PCSI, transactions are not delayed, but they may not observe the latest snapshot. The update transactions' abort rate of PCSI may be higher or lower than that of CSI, depending on system parameters. If the workload is dominated by read-only transactions, PCSI is more suitable than CSI.

\section{Related Work}

Despite the undebatable popularity and practical relevance of CSI, relatively few papers have discussed its properties. CSI was first introduced in 1995 [6, 20]. The authors show that CSI prevents many of the well-known concurrency control anomalies [6], and that CSI is weaker than serializability, e.g., CSI allows some forms of constrain violation anomalies such as write-skew [6] and predicate-writeskew [13]. Schenkel et al. [26] have discussed using CSI in federated databases where global transactions access data that are distributed across multiple sites. Their protocols guarantee CSI at the federation level.

More research is needed for guaranteeing serializability both when using weaker isolation levels and when using ap- 


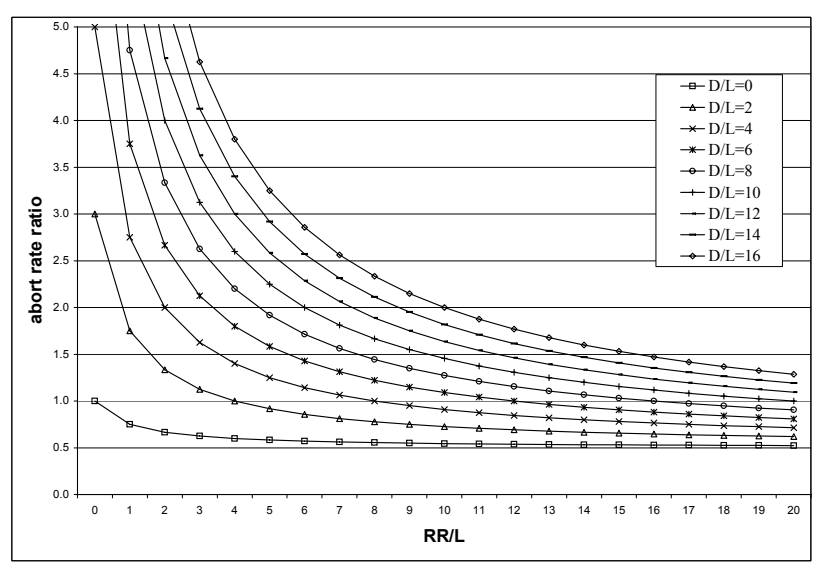

Figure 3. Abort rate ratio of PCSI to CSI in replicated database with centralized certification, parameterized by $D / L$.

plication specific knowledge. Adya et al. [1] have provided a theoretical foundation to formally specify practical isolation levels for commercial databases. Atluri et al. [4] have studied the serializability of weaker isolation levels such as the ANSI SQL isolation levels for centralized databases. Shasha et al. [28] have presented the conditions that allow a transaction to be divided into smaller sub-transactions that release locks earlier than the original transaction under traditional locking policies. Kemme et al. [21] have discussed how to implement different isolation levels (including serializability) in replicated databases using group communication primitives. Two systems, Postgres-R(SI) [31] and Ganymed [24], have been built to replicate databases that use snapshot isolation.

Fekete et al. [11, 13] have studied the conditions under which transactions executing on a centralized database with CSI produce serializable histories. They have developed a syntactic condition similar to condition S1. Condition S1 is an extension to their syntactic condition, and it applies to GSI in centralized and distributed environments. However, their more recent work [12], which is based on analyzing conflict patterns among transactions, identifies necessary and sufficient static conditions for serializability under databases that support snapshot isolation.

Dahlin et al. [15, 14] have discussed application-specific data replication techniques for Web edge services, with the objectives of reducing response time, scaling up system performance, and enhancing its availability. This method is effective but not transparent; application programmers have to specify a replication policy for each "data object". Also, there is no guarantee of serializability. Our methods have the same objectives. However, they are systematic and transparent to application programmers. Serializability of

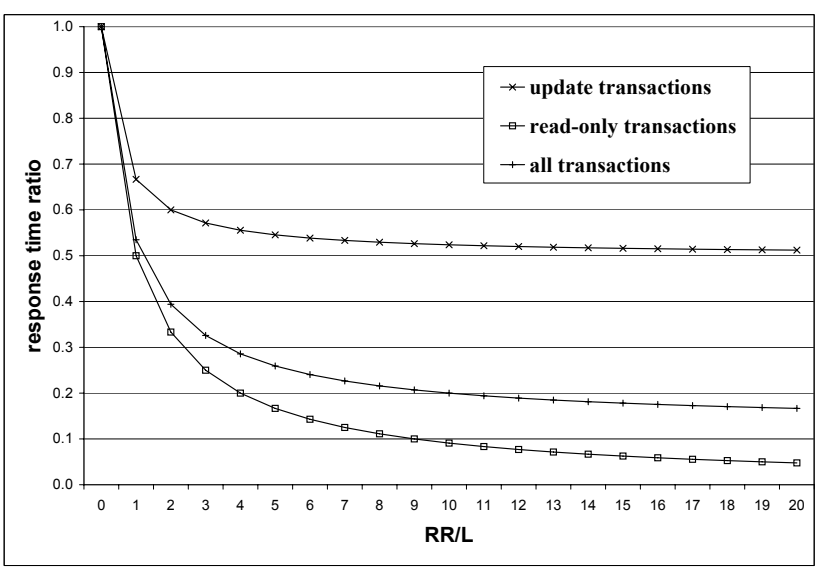

\section{Figure 4. Response time ratio of PCSI to CSI in replicated database with central- ized certification.}

arbitrary transactions can be guaranteed by either rewriting the transactions in the application to satisfy condition S1, or by enforcing condition D3 at run-time.

\section{Conclusions and Future Work}

This research presents generalized snapshot isolation (GSI), which is an extension of conventional snapshot isolation (CSI). GSI does not delay read-only transactions, but they may observe an old snapshot of the database. Serializability under GSI can be guaranteed. We show a dynamic (D3) and a static (S1) condition that make transactions running under GSI produce serializable histories.

We present prefix-consistent snapshot isolation (PCSI), an instance of GSI that is suitable for multi-site replicated databases. PCSI uses the most recent snapshot currently available on a database site, such that each transaction sees the updates of all transactions that have executed and committed at that site. The most important benefits of using PCSI are that it does not delay read-only transactions and executions can be made serializable.

We develop two implementations of PCSI: one uses centralized certification and the other uses distributed certification. We use an analytical model to compare the performance of PCSI to CSI. The model shows that the abort rate of update transactions in PCSI and CSI depends on system parameters and that PCSI may give a lower abort rate than CSI. The model also shows that the response times of both update and read-only transactions are smaller when using PCSI than when using CSI.

We plan to use PCSI in a geographically distributed network of proxies to replicate Web services. Web services are particularly suitable for PCSI: 1- Their workload is dom- 
inated by read-only transactions. 2- Most update transactions are short and have small writesets. 3- The templates of all update transactions are known in advance, which allows using S1 to guarantee serializability.

\section{References}

[1] A. Adya, B. Liskov, and P. E. O'Neil. Generalized isolation level definitions. In Proceedings of the IEEE International Conference on Data Engineering, pages 67-78, 2000.

[2] Akamai Technologies, Inc. EdgeSuite Architecture for Advanced E-Business. http://www.akamai.com/en/ html/services/edgesuite.html.

[3] C. Amza, E. Cecchet, A. Chanda, A. L. Cox, S. Elnikety, R. Gil, J. Marguerite, K. Rajamani, and W. Zwaenepoel. Specification and implementation of dynamic Web site benchmarks. In Proceedings of the 5th Workshop on Workload Characterization, Austin, Texas, November 2002.

[4] V. Atluri, E. Bertino, and S. Jajodia. A theoretical formulation for degrees of isolation in databases. Elsevier Science, 39 No.1, 1997.

[5] G. Ballintijn, M. van Steen, and A. S. Tanenbaum. Characterizing internet performance to support wide-area application development. Operating Systems Review, 34(4):41-47, 2000.

[6] H. Berenson, P. Bernstein, J. Gray, J. Melton, E. O’Neil, and P. O'Neil. A critique of ANSI SQL isolation levels. In Proceedings of the SIGMOD International Conference on Management of Data, pages 1-10, May 1995.

[7] P. Bernstein, V. Hadzilacos, and N. Goodman. Concurrency Control and Recovery in Database Systems. AddisonWesley, Reading, MA, 1987.

[8] E. Cecchet, A. Chanda, S. Elnikety, J. Marguerite, and W. Zwaenepoel. Performance comparison of middleware architectures for generating dynamic Web content. In Proceedings of the ACM/IFIP/USENIX International Middleware Conference (Middleware 2003), Rio de Janeiro, Brazil, June 2003.

[9] Data Concurrency and Consistency, Oracle8 Concepts, Release 8.0: Chapter 23. Oracle corporation. 1997.

[10] S. Elnikety, F. Pedone, and W. Zwaenepoel. Generalized Snapshot Isolation in Replicated Databases. EPFL Technical Report IC/2004/21. http: //ic2.epfl.ch/ labos/gsi.pdf.

[11] A. Fekete. Serialisability and snapshot isolation. In Proceedings of the Australian Database Conference, pages 201-210, Auckland, New Zealand, January 1999.

[12] A. Fekete. Allocating isolation levels to transactions. In ACM conference on Principles of database systems, pages 206-215, Baltimore, MD, June 2005.

[13] A. Fekete, D. Liarokapis, E. O'Neil, P. O'Neil, and D. Shasha. Making snapshot isolation serializable. In $A C M$ transactions on database systems, volume 39(2), pages 492528, June 2005.

[14] L. Gao, M. Dahlin, A. Nayate, J. Zheng, and A. Iyengar. Application specific data replication for edge services. In Proceedings of the twelfth international conference on World Wide Web, pages 449-460. ACM Press, 2003.
[15] L. Gao, M. Dahlin, A. Nayate, J. Zheng, and A. Iyengar. Improving availability and performance with applicationspecific data replication. IEEE Transactions on Knowledge and Data Engineering, 17(1):106-120, Jan 2005.

[16] J. Gray, P. Helland, P. O'Neil, and D. Shasha. The dangers of replication and a solution. In Proceedings of the 1996 ACM SIGMOD International Conference on Management of Data, pages 173-182, June 1996.

[17] J. Gray and A. Reuter. Transaction Processing: Concepts and Techniques. Morgan Kaufmann Publishers, San Francisco, CA, 1993.

[18] V. Hadzilacos and S. Toueg. Fault-Tolerant Broadcasts and Related Problems. Distributed Systems (2nd Ed.), ACM Press / Addison-Wesley Publishing Co., 1993.

[19] InterBase Documentation. Borland Software Corporation. 2004.

[20] K. Jacobs. Concurrency control, transaction isolation and serializability in SQL92 and Oracle7. Technical report, Oracle Corporation, Redwoord City, CA, July 1995. White paper A33745.

[21] B. Kemme and G. Alonso. A suite of database replication protocols based on group communication primitives. In Proceedings 18th International Conference on Distributed Computing Systems (ICDCS), Amsterdam, The Netherlands, May 1998.

[22] C. Papadimitriou. The Theory of Database Concurrency Control. Computer Science Press, 1986.

[23] F. Pedone, R. Guerraoui, and A. Schiper. The database state machine approach. Distributed and Parallel Databases, (14):71-98, 2003.

[24] C. Plattner and G. Alonso. Ganymed: Scalable Replication for Transactional Web Applications. In Proceedings of the ACM/IFIP/USENIX International Middleware Conference (Middleware 2004), Toronto, Canada, October 2004.

[25] PostgreSQL 7.4 Documentation. PostgreSQL Global Development Group. 2004.

[26] R. Schenkel, G. Weikum, N. Weißenberg, and X. Wu. Federated transaction management with snapshot isolation. Lecture Notes in Computer Science, 1773:1-25, January 2000.

[27] F. Schneider. Replication Management using the StateMachine Approach. Distributed Systems (2nd Ed.), ACM Press / Addison-Wesley Publishing Co., 1993.

[28] D. Shasha, F. Llirbat, E. Simon, and P. Valduriez. Transaction chopping: Algorithms and performance studies. ACM Transactions on Database Systems, 20(3):325-363, 1995.

[29] The Transaction Processing Council (TPC). Full disclosure report for Dell PowerEdge 6650 1.6GHz w/PowerEdge 1650 1.4GHz. http://www.tpc.org/results/ FDR/tpcw/PE6650_10K_TPCW_FDR_020822.pdf.

[30] The Transaction Processing Council (TPC). The TPC-C and TPC-W Benchmarks. http: //www.tpc.org/.

[31] S. Wu and B. Kemme. Postgres-R(SI): Combining replica control with concurrency control based on snapshot isolation. In International Conference of Data Engineering, 2005.

[32] Yukon Release Microsoft SQL Server. Microsoft Corporation. 2005. 